\title{
Proteomic signature of periodontal disease in pregnancy: Predictive validity for adverse outcomes
}

\author{
Manisha Ramchandani ${ }^{1}$, Muniza Siddiqui ${ }^{1}$, Raveena Kanwar ${ }^{1}$, Manwinder \\ Lakha $^{1}$, Linda Phi ${ }^{1}$, Luca Giacomelli ${ }^{2}$, Francesco Chiappelli ${ }^{1}$
}

${ }^{1}$ Division of Oral Biology \& Medicine, UCLA School of Dentistry, CHS 63-090, Los Angeles, CA 90095-1668; ${ }^{2}$ Institute for Stomatology Research, Lido di Camaiore, Italy; Manisha Ramchandani- Email: rmanisha@ucla.edu; *Corresponding author

Received November 11, 2010; Accepted November 17, 2010; Published January 06, 2011

\begin{abstract}
:
The rate of preterm birth is a public health concern worldwide because it is increasing and efforts to prevent it have failed. We report a Clinically Relevant Complex Systematic Review (CSCSR) designed to identify and evaluate the best available evidence in support of the association between periodontal status in women and pregnancy outcome of preterm low birth weight. We hypothesize that the traditional limits of research synthesis must be expanded to incorporate a translational component. As a proof-of-concept model, we propose that this CSCSR can yield greater validity of efficacy and effectiveness through supplementing its recommendations with data of the proteomic signature of periodontal disease in pregnancy, which can contribute to addressing specifically the predictive validity for adverse outcomes. For this CRCSR, systematic reviews were identified through The National Library of MedicinePubmed, The Cochrane library, CINAHL, Google Scholar, Web of Science, and the American Dental Association web library. Independent reviewers quantified the relevance and quality of this literature with R-AMSTAR. Homogeneity and inter-rater reliability testing were supplemented with acceptable sampling analysis. Research synthesis outcomes were analyzed qualitatively toward a Bayesian inference, and converge to demonstrate a definite association between maternal periodontal disease and pregnancy outcome. This CRCSR limits heterogeneity in terms of periodontal disease, outcome measure, selection bias, uncontrolled confounders and effect modifiers. Taken together, the translational CRCSR model we propose suggests that further research is advocated to explore the fundamental mechanisms underlying this association, from a molecular and proteomic perspective.
\end{abstract}

Keywords: proteomic signature of periodontal disease, pregnancy, adverse pregnancy outcome, pregnancy complications, preterm, systematic review, clinically relevant complex systematic review (CSCSR), meta-analysis

\section{Background:}

Periodontal disease, including gingivitis and periodontitis, is among the most common chronic disorders of infectious origin in humans. The American Academy of Periodontology reported in 2004 a prevalence range for this condition between 10 to $60 \%$ in adults, depending on diagnostic criteria, with a 30-90\% overall across age, gender and ethnicity prevalence range in the United States. The national health and nutrition examination survey 111(NHANES 111) confirmed that over $90 \%$ of individuals above 13 years of age present some form of periodontal disease.

Periodontal disease is associated with certain systemic conditions as increased risk of cardiovascular and cerebrovascular diseases, including atherosclerosis and diabetes [1]. Cardiovascular disease has complex determining factors, including genetics and gender, but evidence shows that chronic periodontal infection is detrimental to the cardiovascular system. Several theories have been proposed to explain the pathway between oral infection and cardiovascular complications [1]. One is that chronic infection of the mouth may lead to thromboembolic disease (blood clotting) via bacterial protein interfering with platelet aggregation [2]. Another possibility is that bacteria from the mouth enter the bloodstream and trigger the production of pro-inflammatory cytokines, which are responsible for promoting inflammation and over time lead to pathology of the cardiovascular system. ${ }^{3}$ Respiratory infections have also been linked to periodontitis [1].
The rate of preterm birth is increasing worldwide while efforts to prevent or reduce its prevalence have been unsuccessful [4]. Developing countries report high incidences of low birth weight; and, in industrialized countries, prematurity rates have ceased to fall since the 1980s, and even shown a consistent rising trend. Taken together, these data point to an alarming situation of major social and economic public health concern worldwide [5].

Approximately 500,000 infants (over $12 \%$ of live births) were delivered preterm (gestational age $<37$ weeks) in 2003, which signifies a $16 \%$ increase since 1990, in the US [6]. Preterm birth is the leading cause of neonatal mortality and morbidity worldwide. Preterm birth and low birth weight, which often occur together, are risk factors for certain serious lifelong health problems, from blindness, deafness, developmental delay, heart disease, chronic lung disease, to a range of neurological disorders. Health care costs associated with preterm birth are substantial both prenatally and into adulthood [9]. Preventing preterm birth and low birth weight is the most timely, critical, and effective approach to avoid and prevent these lifelong health conditions [7].

Risk factors of adverse pregnancy potentially leading to preterm birth and low birth weight include previous history of prematurity, placental abnormalities, smoking, diabetes, gestational weight gain, vaginal 
infections, and oral health. Whereas certain risk factors associated with preterm birth and low birth weight are non-modifiable factors (e.g., higher incidence of multiple births and advanced maternal age (Health Canada)), others can be controlled. Case in point, approximately $50 \%$ of preterm births occur without a known cause, of which up to half may be attributed to maternal bacterial infections [8].

The putative link between periodontitis and adverse pregnancy outcomes was proposed by Galloway (1931). Galloway suggested that Gramnegative anaerobic bacteria might "provide sufficient infectious microbial challenge [to have] potentially harmful effects on the pregnant patient and developing fetus" [10]. This hypothesis has been supported by a growing consensus that preterm birth and low birth weight may be influenced by chronic bacterial infections [1], and specifically that bacteria from the oral cavity enter the bloodstream and affect the placenta and amniotic fluid. The bacteria in the placenta and amniotic fluid trigger an inflammatory response, which can, under appropriate conditions that require the release of prostaglandins, induce preterm labor, or, in early pregnancy, miscarriage. Copious evidence now demonstrates that normal induction of labor occurs when the fetus has reached complete gestation, but with maternal periodontitis during pregnancy, prostaglandin release significantly increases the risk of preterm labor [8]. Furthermore, periodontitis, as a low-grade chronic infection, may also be blunt growth of the fetus, and lead to low birth weight [8]

Taken together, the copious evidence at hand strongly suggests a strong association between periodontal diseases in pregnant women and increased risk of adverse pregnancy outcome. This study is aimed at utilizing the research synthesis design to provide a clinically relevant complex systematic review (CRCSR) of the best available evidence on the association between periodontal status in women and pregnancy outcome preterm low birth weight. We also propose a hypothesis about how the clinical relevance of the CRCSR findings may be broadened by a translational arm addressing the proteomic signature of periodontal disease in pregnancy, in order to yield novel understanding of the predictive variables for adverse pregnancy outcomes.

\section{Methodology:}

To conduct this complex systematic review, we utilized a research synthesis design as follows: the PICO question was stated as "is there an association between maternal periodontal disease and preterm low birth weight infants from which flowed the search protocol and strategy, and inclusion/exclusion criteria, the quantification of the quality of evidence, and homogeneity, reliability and acceptable sampling analyses.” The clinical relevance of the consensus statement was estimated, and clinical recommendations formulated.

Specifically, the search used electronic databases, The National Library of Medicine-Pubmed, The Cochrane library, CINAHL, Google Scholar, Web of Science, and the American Dental Association web library to identify systematic reviews on the association of maternal periodontal disease and preterm birth and low birth weight. Inclusion criteria included published systematic reviews and meta-analysis on the risk of adverse pregnancy outcome and maternal periodontal disease. Exclusion criteria included traditional reviews, theses, dissertations, and conference proceedings, reviews that were neither published nor available online and non peerreviewed articles. Selection of reviews was performed based on the criteria for considering systematic reviews for inclusion. The data were recorded onto a extraction sheet as part of the CRCSR: study design, diagnosis, number of patients, types of intervention, outcome measures, results, quality score, and author's conclusion. The reviewers evaluated the methodological quality of each using the R-AMSTAR [12]. R-AMSTAR also produces a quantifiable assessment of systematic review quality and clinical relevance. All the articles were discussed until consensus was reached. The inter-rater reliability was calculated with Cohen's kappa coefficient. Homogeneity was tested by means of the Chi-square analysis. Acceptable sampling analysis was performed using the Friedman test for non-parametric analysis of factorial designs using MDAS statistical software.

\section{Discussion:}

The search term 'pregnancy' resulted in 681977 citations and there were 64193 results with periodontal disease. They were reduced to 1027 citations. After the initial screen, 1016 citations were excluded since they did not meet the inclusion criteria. Of the 11 potentially relevant citations, two were literature reviews, one was a correspondence to a review, one was an editorial, and one review was in Polish. Therefore, these five reviews were not included. Finally, using the inter reliability testing as well as R-AMSTAR [12], 6 systematic reviews were obtained by this systematic search and used for this Clinically Relevant Complex Systematic Review (CRCSR). The Friedman test was done for nonparametric analysis of factorial designs. Each systematic review was tested to see if it was statistically significant ( $p$-value $=0.0032$ ). Each review was then compared of their individual means and standard deviations. The results of the acceptable sampling analysis based on R-AMSTAR scoring of included systematic reviews are shown in Table 1 (see Supplementary material). The horizontal rows indicate each included systematic review. The vertical column indicates the eleven question items on the RAMSTAR. The highest scoring systematic review is with 35.5 points, while the lowest scoring systematic review is with 20.5 points [13]. This gives us an indication of the quality of the included systematic reviews within the domain of this association.

The results of two of the systematic reviews, Vergnes et al. (2007) [5] and Khader and Ta'ani (2005) [14], showed relation that lead to the consensus statement that there is an association between maternal periodontal disease and adverse pregnancy outcomes. However, methodological weaknesses of the systematic reviews conducted to date and the individual studies included in them render this conclusion tentative, hence more research should be conducted. In particular, we believe that the identification of genomic and proteomic markers may represent an added value in the further investigation of the association between periodontitis and adverse pregnancy outcomes, as previously suggested [15]. Theoretical studies have investigated the molecular mechanisms underlying periodontitis, and led to the identification of some candidate genes and/or proteins which can become the targets of ad hoc theoretical studies [16]. Similar studies have investigated the association between periodontitis and diabetes [17]. In another recent analysis, a proper combination of experimental and theoretical findings was applied to provide further insights into the malignant progression from oral lichen planus to oral carcinoma [15]. We are presently pursuing a similar approach to increase our knowledge of the adverse pregnancy outcomes in periodontal patients at a molecular level. This analysis study can suggest new potential risk factors and therapeutic targets.

\section{Conclusion:}

There is growing evidence that there is an association between maternal periodontal disease and adverse pregnancy outcome, in particular preterm low birth weight. While researchers would move towards a deeper analysis of the consistent and strong association between periodontal disease and adverse pregnancy outcomes, the decision makers like the clinicians, policy makers and the patients should be aware of this emerging evidence and should appreciate the role of maternal oral health during pregnancy. Many guidelines have been established "to assist health care professionals in improving clinical practice and to promote oral health in pregnant women” by the New York State Health Department [21] It is essential that women get the needed treatment that is provided by their dentist during the period between 14th and 20th week of their pregnancy, and get regular checkups throughout the course. It is also optimal to have an adequate diet: the appropriate use of fluoride, eating fruits and drinking water will encourage a smaller amount of sugar intake and thus prevent from building up plaque and cavities in the mouth. Good oral hygiene should be adopted by brushing and flossing twice daily. Expectant women are prone to get bacterial transmission that promotes tooth decay through any saliva exchange, and thus should avoid sharing food or utensils with others. Oral health education triggers significant health benefits while also preventing complications of dental diseases during pregnancy and thus reducing the chances of low birth weight deliveries. Note that the guidelines are not limited to the ones mentioned above. Therefore, maintaining good oral 


\section{Bioinformation}

health care should be highly encouraged for women who are pregnant or wish to become pregnant.

\section{References:}

[1] YT Teng. Infect. Imm. 70: 5269 (2002) [PMID: 12183580]

[2] HA Schenkein et al. Periodontol. 45: 113 (2007) [PMID: 17850452]

[3] PJ Pussinen et al. Arterioscler. Thromb. Vasc. Biol. 27: 1433 (2007) [PMID: 17363692]

[4] G Wimmer \& BL Pihlstrom, J. Clin. Periodontol. 35S: 380 (2008) [PMID: 18724864]

[5] JN Vergnes \& M Sixou, Am. J. Obstetrics Gynecol. 196: 135e1

[6] RC Page et al. J Clin Periodontol. 30: 819 (2003) [PMID: 12956658]

[7] M Hack \& AA Fanaroff. Early Human Develop. 53: 193(1999) [PMID: 10088988]

[8] M Wener \& S Lavigne, Association of Women's Health, Obstetric and Neonatal Nurses Lifeline 8: 422 (2004) [PMID:15560620]

[9] GS Berkowitz \& E Papiernik, Epidemiol. Rev. 15: 414 (1993) [PMID: 8174665]

[10] M Radnai et al. J. Clin. Periodontol. 31: 736 (2004) [PMID: 15312095] (2007) [PMID: 17306654]

[11] YA Bobetsis et al. J. Am. Dent. Assoc. 137S: 7 (2006) [PMID: 17012730]

[12] J Kung et al. The Open Dent. J. 4: 84 (2010) [PMID: 21088686]

[13] MV Vettore et al. Caderno de Saude Publica. 40: 184 (2006) [PMID: 16951876]

[14] YS Khader \& Q Ta'ani, J Periodonto, 76: 161 (2005) [PMID: 15974837]

[15] L Giacomelli \& U Covani. Open Dent J. 4: 67 (2010) [PMID: 20871759]

[16] KM Hettne et al. J. Clin. Periodonto. 34: 1016 (2007) [PMID: 18028194]

[17] U Covani et al. J. Periodonto. 79: 1974 (2008) [PMID: 18834254]

[18] U Covani et al. J. Periodontol. 80: 1265 (2009) [PMID: 19656026]

[19] OO Oluwadara et al. Bioinformation 4: 249 (2009) [PMID: 20975919]

[20] L Giacomelli et al. Bioinformation 4: 258 (2009) [PMID: 20975920]

[21] A Samelson et al. Oral health care during pregnancy and early childhood: practice guidelines. Albany: New York State Department of Health. (2006) 5. http:// www.health.state.ny.us/ publications/ 0824.pdf.

Edited by F Chiappelli

Citation: Ramchandani et al. Bioinformation 5(7): 300-303 (2011) License statement: This is an open-access article, which permits unrestricted use, distribution, and reproduction in any medium, for non-commercial purposes, provided the original author and source are credited. 


\section{Bioinformation Volume 5}

\section{Supplementary material:}

Table 1: Mean scores given to each question of the eleven questions of the R-AMSTAR for the six included systematic reviews.

\begin{tabular}{|c|c|c|c|c|c|c|c|c|c|c|c|c|c|c|}
\hline \multicolumn{15}{|c|}{\begin{tabular}{|c|} 
Acceptable Sampling Analysis based on R-AMSTAR \\
Scores shown are the mean of the individual scores of the two independent readers
\end{tabular}} \\
\hline & 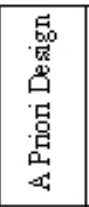 & 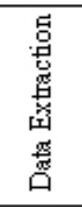 & 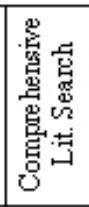 & 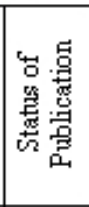 & 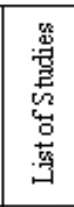 & 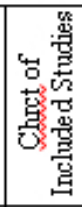 & 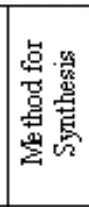 & 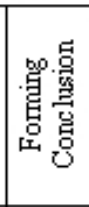 & 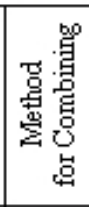 & 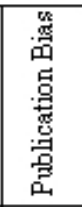 & 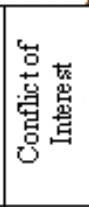 & & & \\
\hline & 1 & 2 & 3 & 4 & 5 & 6 & 7 & 8 & 9 & 10 & 11 & Total & Mean & SD \\
\hline $\begin{array}{c}\text { Vergnes et al, } \\
2007\end{array}$ & 3.5 & 3.5 & 3.5 & 3.5 & 3.5 & 2.5 & 3 & 3 & 4 & 4 & 1.5 & 35.5 & 3.23 & 0.72 \\
\hline Xiong et al, 2007 & 2 & 1.5 & 3 & 1 & 1 & 3 & 2.5 & 2 & 3 & 1 & 1 & 21 & 1.19 & 0.86 \\
\hline $\begin{array}{l}\text { Vettore et al, } \\
2006\end{array}$ & 3 & 1.5 & 3 & 1 & 1 & 2 & 1 & 4 & 2 & 1 & 1 & 20.5 & 1.86 & 1.05 \\
\hline Xiong et al, 2006 & 3 & 1.5 & 2 & 1 & 1 & 2 & 3 & 3 & 4 & 1 & 1 & 22.5 & 2.05 & 1.06 \\
\hline $\begin{array}{l}\text { Khader and } \\
\text { Ta'ani, } 2003\end{array}$ & 3 & 4 & 4 & 1.5 & 2.5 & 1.5 & 1.5 & 3 & 4 & 4 & 2 & 31 & 2.82 & 1.08 \\
\hline $\begin{array}{c}\text { Scannapieco et } \\
\text { al, 2003 }\end{array}$ & 3 & 3 & 2 & 1.5 & 2 & 1 & 2 & 2 & 2.5 & 1 & 1 & 21 & 1.91 & 0.74 \\
\hline Total & 17.5 & 15 & 17.5 & 9.5 & 11 & 12 & 13 & 17 & 19.5 & 12 & 7.5 & & & \\
\hline Mean & 2.92 & 2.50 & 2.92 & 1.58 & 1.83 & 2.00 & 2.17 & 2.83 & 3.25 & 2.00 & 1.25 & & & \\
\hline SD & 0.49 & 1.14 & 0.80 & 0.97 & 1.03 & 0.71 & 0.82 & 0.75 & 0.88 & 1.55 & 0.42 & & & \\
\hline
\end{tabular}

